Check for updates

Cite this: RSC Adv., 2019, 9, 28561

Received 13th July 2019

Accepted 26th August 2019

DOI: $10.1039 / c 9 r a 05371 a$

rsc.li/rsc-advances

\section{Beneficial effects of potassium iodide incorporation on grain boundaries and interfaces of perovskite solar cells $\uparrow$}

\author{
Yin Yang, ${ }^{a}$ Lili Wu, ${ }^{\star a b}$ Xia Hao, (D) ${ }^{* b}$ Zeguo Tang, ${ }^{c}$ Huagui Lai, ${ }^{a}$ Jingquan Zhang, ${ }^{\text {ab }}$ \\ Wenwu Wang ${ }^{a}$ and Lianghuan Feng ${ }^{a}$
}

Grain boundaries and interfacial impurities are the main factors that limit the further development of polycrystalline perovskite solar cells because their existence severely deteriorates the device performance. In order to optimize the efficiency of perovskite solar cells, it is essential to eliminate these defects. In the present work, potassium iodide $(\mathrm{KI})$ is incorporated into the perovskite absorber. $\mathrm{KI}$ incorporation improves the crystallinity of the perovskite, increases the grain size, and decreases the contact potential distribution at the grain boundary, which are verified by X-ray diffraction, scanning electronic microscopy and Kelvin probe force microscopy. Besides, the activation energy of the recombination, estimated from the temperature dependent current-voltage of perovskite solar cells, is larger than the bandgap calculated from the temperature coefficient. These suggest that $\mathrm{KI}$ incorporation effectively passivates the grain boundaries and interfacial defects. As a result, charge trapping in the absorber as well as the bimolecular and trap-assisted recombination of the device are significantly suppressed. Consequently, the open circuit voltage and fill factor of the incorporated devices are greatly improved, enabling an optimized power conversion efficiency of $19.5 \%$, in comparison with that of $17.3 \%$ for the control one. Our work provides an effective strategy of defect passivation in perovskite solar cells by $\mathrm{KI}$ incorporation and clarifies the mechanism of the performance optimization of $\mathrm{Kl}$ incorporated devices.

\section{Introduction}

Over the past decade, the efficiency of organic-inorganic halide hybrid perovskite solar cells has increased from $3.8 \%$ to over $24 \%$. Such rapid development has made them a strong competitor in the thin film solar cell community. ${ }^{1}$ One of the most important reasons for the wide investigation of perovskite solar cells is the advantages of the organic-inorganic halide hybrid perovskite material, such as long lifetime and diffusion length of the carrier, ${ }^{2-4}$ the tuneable band gap. ${ }^{5}$ Besides, the inexpensive raw materials and simple preparation processes

anstitute of Solar Energy Materials and Devices, College of Materials Science and Engineering, Sichuan University, Chengdu 610064, China. E-mail: wulili@scu.edu. cn; hao.xia0808@scu.edu.cn; Fax: +8628 85412542; Tel: +862885412542

${ }^{b}$ Institute of New Energy and Low-Carbon Technology, Sichuan University, Chengdu 610027, China

'Chengdu Research Center, Hanergy Thin Film Power Group Ltd., Chengdu 610200, China

$\dagger$ Electronic supplementary information (ESI) available: $J-V$ curves of five control and incorporated devices; the absolute values of $J_{\mathrm{sc}}, V_{\mathrm{oc}}, \mathrm{FF}$ and PCE obtained from the degradation process of control and KI incorporated devices; JVT curves of control and KI incorporated devices during heating and cooling process. See DOI: $10.1039 / \mathrm{c} 9 \mathrm{ra05371a}$ make it possible to achieve large-scale and cost-effective production.

The common structure of the organic-inorganic halide hybrid perovskite material is $\operatorname{AMX}_{3}(\mathrm{~A}=$ methylammonium (MA) $\mathrm{CH}_{3} \mathrm{NH}_{3}{ }^{+}$, or formamidinium (FA) $\mathrm{CH}_{3}\left(\mathrm{NH}_{2}\right)_{2}{ }^{+} ; \mathrm{M}=\mathrm{Pb}^{2+}$ or $\mathrm{Sn}^{2+} ; \mathrm{X}=\mathrm{Cl}^{-}, \mathrm{Br}^{-}$or $\mathrm{I}^{-}$). The solution-processable spin coating is one of the regular techniques for preparing absorber and other functional layers of perovskite solar cells. However, impurities at the grain boundaries (GBs) and interfacial defects are inevitable. Although GBs are not acting as recombination centres, they severely hinder the lateral diffusion of carriers and as a result, degrade the device performance. ${ }^{6}$ Thus, there have been many reports attempting to modify GBs by incorporating various additives into the absorption layer of perovskite solar cells to achieve improvements both in efficiency and in stability. A passivation functional groups contained carboxyl, amine groups and aromatic structures were customized by Yang et al., and the loss of open circuit voltage for the perovskite solar cell was reduced due to the successful elimination of defects in the absorption layer. ${ }^{7}$ Niu et al. demonstrated that the trap states near the GB can be effectively passivated by semiconductor molecules having Lewis acid functional groups. ${ }^{8}$ In addition, interfacial defects are generated during the fabrication process of a sandwich-structured perovskite solar cells, they deteriorate 
the device performance due to the increased trap-assisted recombination probabilities and recombination velocities. The possible strategy could be modifying the contact properties between the perovskite layer and functional layers by incorporating additives into absorber. Lee et al. added phenethyl ammonium to the absorption layer to form a quasi-twodimensional material with a large band gap at the interface, which provided effective interface passivation and an optimized device performance was witnessed. ${ }^{9}$

Alkali metals such as $\mathrm{K}^{10,11} \mathrm{Rb}^{12,13}$ and $\mathrm{Cs}^{14,15}$ with small ionic radii are often used to replace part of the A-site organic ion of the perovskite structure and the incorporation of alkali metals into perovskites has been proved to be beneficial to suppress the hysteresis effect and improve the stability of perovskite solar cells. It has been confirmed that the incorporating of KI will improve the overall performance of perovskite solar cells. The results of Tang et al. suggested that the band alignment of the perovskite could be modified by KI incorporation, and the electron transport barrier was then reduced. The hysteresis effect was therefore eliminated. ${ }^{11} \mathrm{Bu}$ et al. found that KI could eliminated hysteresis by enhancing conductivity which accelerated the charge transport and distribution. ${ }^{16}$

In our work, we investigated the effects of the defects at GBs and interfaces from the point view of charge transportation and recombination. We found that KI incorporation realized an obvious increase in PCE of perovskite solar cells and the shelf lifetime $\tau_{80}$ of the unencapsulated devices increased from less than 200 hours to over 1000 hours. The Kelvin probe force microscopy (KPFM), steady state/time-resolved photoluminescence (PL) and temperature-dependent current density-voltage (JVT) measurements were carried out to clarify the mechanism of performance improvement induced by $\mathrm{KI}$ incorporation. The results suggest that the main reason of these improvements is the effective GB and interface passivation, which decrease the barrier of charge transport at the GBs of perovskites and suppress the bimolecular and trap-assisted recombination of the solar cells. This verifies the importance of GB and interface passivation for optimizing both the power conversion efficiency and the long-term stability of perovskite solar cells.

\section{Experiment}

\section{Device fabrication}

The structure of the perovskite solar cells in this work is glass/ $\mathrm{FTO} / \mathrm{SnO}_{2} /$ compact-TiO $/$ mesoporous- $\mathrm{TiO}_{2} /\left(\mathrm{FA}_{0.83} \mathrm{MA}_{0.17}\right)$ $\mathrm{Pb}\left(\mathrm{I}_{0.83} \mathrm{Br}_{0.17}\right)_{3} /$ spiro-OMeTAD/Au. The FTO-coated glass substrates (NSG TEC 12D, with a $12 \Omega \square^{-1}$ square resistance) were purchased from Pilkington Group Limited and a $30 \mathrm{~nm}$ thick $\mathrm{SnO}_{2}$ was then sputtered. The substrates were cleaned in sequence in an ultrasonic bath with Amway, deionized water and ethanol. After being dried with $\mathrm{N}_{2}$, the substrates were finally treated by UV-ozone for 15 minutes. The titanium diisopropoxide diluted in absolute ethanol was used to prepare the compact- $\mathrm{TiO}_{2}\left(\mathrm{c}-\mathrm{TiO}_{2}\right)$ by spray pyrolysis with $\mathrm{N}_{2}$ as a carrier gas on a hot plate at $400{ }^{\circ} \mathrm{C}$. A mesoporous $\mathrm{TiO}_{2}\left(\mathrm{mp}-\mathrm{TiO}_{2}\right)$ layer was then deposited by spin coating for $40 \mathrm{~s}$ at $4000 \mathrm{rpm}$ with a ramp of $2000 \mathrm{rpm} \mathrm{s}^{-1}$ to achieve a 150-200 nm-thick layer, using a $\mathrm{TiO}_{2}$ particle paste (Dyesol 30 NR-D) diluted in ethanol.

The $\left(\mathrm{FA}_{0.83} \mathrm{MA}_{0.17}\right) \mathrm{Pb}\left(\mathrm{I}_{0.83} \mathrm{Br}_{0.17}\right)_{3}$ film was formed by onestep spin coating of the mixed perovskite precursor solution. The composition of the perovskite precursor solution was FAI ( 1 $\mathrm{M}), \mathrm{PbI}_{2}(1.05 \mathrm{M}), \mathrm{MABr}(0.2 \mathrm{M})$ and $\mathrm{PbBr}_{2}(0.2 \mathrm{M})$ in a mixed solvent of anhydrous $N, N$-dimethylformamide (DMF) and dimethyl sulfoxide (DMSO). The KI incorporated perovskites were obtained by adding $1.5 \mathrm{M}$-KI solution into precursors without introducing any other chemicals.

To deposit a perovskite film, the perovskite precursor solution was transferred to an $\mathrm{N}_{2}$ glove box with a relative humidity less than $10 \%$, and then dropped onto the $\mathrm{TiO}_{2}$-coated FTO substrate. The substrate was spun at $1000 \mathrm{rpm}$ and $4000 \mathrm{rpm}$ for $10 \mathrm{~s}$ and $50 \mathrm{~s}$ respectively. $300 \mu \mathrm{L}$ of chlorobenzene was poured on the substrate $10 \mathrm{~s}$ prior to the end of the program. The asspun perovskite film was then annealed on a hot plate at $160{ }^{\circ} \mathrm{C}$ for 15 minutes. The hole transport layer was spin-coated on the surface of perovskite layer at $3000 \mathrm{rpm}$ for $30 \mathrm{~s}$ by dropping a solution of spiro-OMeTAD in chlorobenzene (72.3 $\mathrm{mg} / 1 \mathrm{~mL}$ ) incorporated by lithium bis(trifluoromethylsulphonyl)imide (Li-TFSI, Sigma-Aldrich) and 4tert-butylpyridine (TBP, Sigma-Aldrich). Finally, a 100 nm-thick Au electrode was thermally evaporated via a shadow mask and the full structure of the solar cell with a typical area of 0.1886 $\mathrm{cm}^{2}$ was completed.

\section{Characterization}

The X-Ray Diffraction (XRD) was performed on a DX-2600 X-ray diffractometer (Dandong Fangyuan Instrument Company) with $\mathrm{Cu}-\mathrm{K} \alpha$ radiation (scan range from $10^{\circ}$ to $70^{\circ}$ ). The morphology of the film was observed by a field emission scanning electron microscope (Hitachi S-4300). KPFM measurement was performed on the surface of the perovskite films in ambient air condition, using Multi Mode 8 AFM with commercially available MESP conductive probe coated with $\mathrm{Co} / \mathrm{Cr}$. KPFM is used to study the spatially resolved imaging of surface contact potential difference (CPD). The photovoltaic characteristics were measured using a Keithley 2400 Source Meter under simulated AM 1.5G sunlight (Sun 2000 solar simulator, ABET Technology) with an illumination power density of $100 \mathrm{~mA} \mathrm{~cm}{ }^{-2}$, which is calibrated by a GaAs standard cell. The JVT characteristics of solar cells were carried out under a simulated AM1.5 sunlight. The light-induced temperature gradient on the sample was negligible and the temperature was increased from $300 \mathrm{~K}$ to 360 $\mathrm{K}$ with a step of $5 \mathrm{~K}$. The device was heated by a self-made resistance heater and the temperature was monitored by a bonded thermocouple (ST-50, PKC, Inc.). The steady state photoluminescence (PL) and time-resolved photoluminescence (TRPL) of perovskite films were characterized by the Edinburgh Instruments FLS 980 fluorescence spectrometer.

\section{Results and discussion}

The cell structure is glass $/ \mathrm{FTO} / \mathrm{SnO}_{2} / \mathrm{c}-\mathrm{TiO}_{2} / \mathrm{mp}-\mathrm{TiO}_{2} /$ $\mathrm{K}\left(\mathrm{FA}_{0.83} \mathrm{MA}_{0.17}\right) \mathrm{Pb}\left(\mathrm{I}_{0.83} \mathrm{Br}_{0.17}\right)_{3} /$ spiro-OMeTAD/Au. The volume 
ratio of $\mathrm{KI}$ is set as $0 \%\left[\mathrm{~K}_{0}\left(\mathrm{FA}_{0.83} \mathrm{MA}_{0.17}\right) \mathrm{Pb}\left(\mathrm{I}_{0.83} \mathrm{Br}_{0.17}\right)_{3}\right], 3 \%$ $\left[\mathrm{K}_{0.03}\left(\mathrm{FA}_{0.83} \mathrm{MA}_{0.17}\right)_{0.97} \mathrm{~Pb}\left(\mathrm{I}_{0.83} \mathrm{Br}_{0.17}\right)_{3}\right]$ and $6 \% \quad\left[\mathrm{~K}_{0.06}\left(\mathrm{FA}_{0.83}{ }^{-}\right.\right.$ $\left.\left.\mathrm{MA}_{0.17}\right)_{0.94} \mathrm{~Pb}\left(\mathrm{I}_{0.83} \mathrm{Br}_{0.17}\right)_{3}\right]$, which are marked as control, $3 \% \mathrm{~K}^{+}$ and $6 \% \mathrm{~K}^{+}$respectively (the volume ratio is defined as $M_{\mathrm{KI}} / M_{\mathrm{KI}}+$ $\left.M_{\mathrm{FA}}+M_{\mathrm{MA}}\right)$. The photovoltaic parameters are obtained from 10 units cell of each batch, as shown in Fig. 1. Best performance is achieved when the molar ratio of KI is $3 \%$ and the average parameters are: short circuit current density $\left(J_{\mathrm{sc}}\right)=24.67 \mathrm{~mA}$ $\mathrm{cm}^{-2}$, open circuit voltage $\left(V_{\mathrm{oc}}\right)=1.07 \mathrm{eV}$, fill factor $(\mathrm{FF})=$ $72.86 \%$, power conversion efficiency $(\mathrm{PCE})=19.27 \%$.

The short-circuit current density decreased when we kept increasing the volume ratio of KI to $6 \%$. It has been clarified that this performance deterioration was mainly due to the lattice constant mismatch caused by $\mathrm{K}^{+}$with a small ionic radius $(1.33 \AA)$. It is kinetically unfavourable for films crystallization to produce a preferred face orientation. ${ }^{17}$ Therefore, in this study, we chose $3 \%$ as the incorporating concentration of KI for further investigation.

The morphologies of the perovskite films are shown in Fig. 2. After KI incorporation, the grain size of the film is obviously increased, as shown in Fig. 2a and c. One may find in Fig. 2e that after KI incorporation, the average grain size increased from 0.64 to $1.07 \mu \mathrm{m}$, and half of grains grew to over $1 \mu \mathrm{m}$. Therefore, the GBs were significantly reduced after KI incorporation, which can effectively suppress the effect of ion diffusion between the functional layers of the device. It is worth noting that in Fig. $2 \mathrm{~b}$ and $\mathrm{d}$, the large grains are perpendicular to the substrate, which suggests that here only the vertical GBs are formed. Therefore, in case of the KI incorporated devices, the vertical GBs decreased, and no extra lateral GBs were introduced. Therefore, the charge trapping interfaces and photocurrent conduction paths in the KI incorporated devices will be effectively decreased. ${ }^{18}$ Besides, by investigating series of SEM images for control and $\mathrm{K}^{+}$incorporated perovskite films, we found that after KI incorporation, small particles were emerged at the GBs, as observed in Fig. 2c. It has been confirmed that these particles are $\mathrm{PbI}_{2}$. Their results also suggest that the
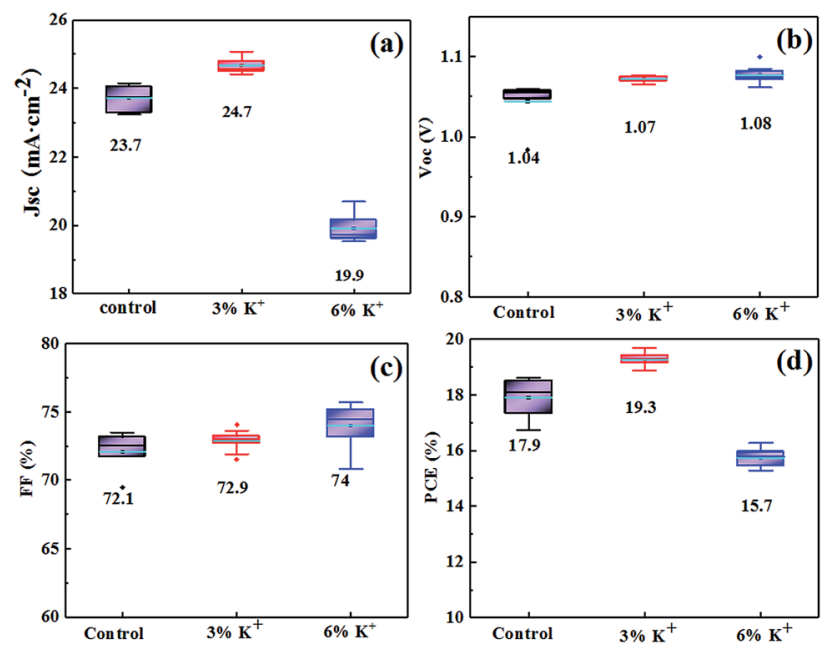

Fig. 1 The box charts of performance parameters: (a) $J_{\mathrm{sc}}$ (b) $V_{\mathrm{oc}}$, (c) FF and (d) PCE for perovskite solar cells with different incorporating concentrations.

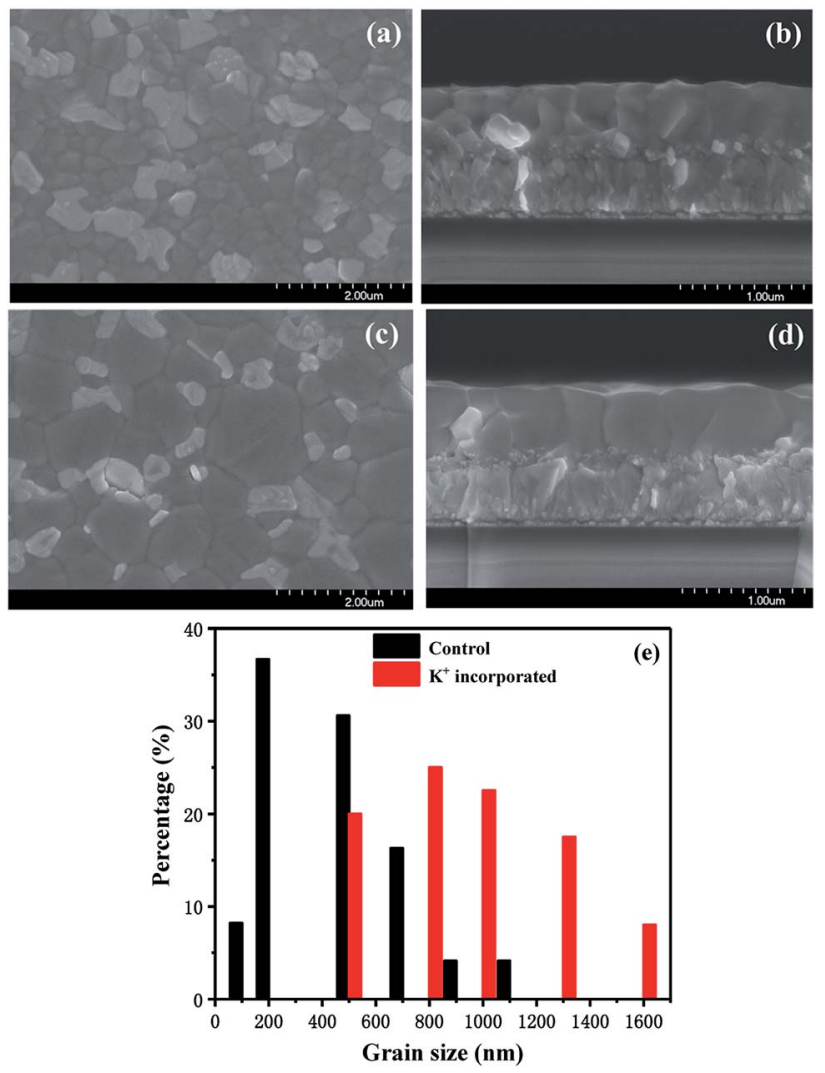

Fig. 2 Surface and cross-sectional morphologies of (a and b) control and (c and d) $\mathrm{K}^{+}$incorporated perovskite films. (e) Grain size distribution corresponding to SEM images.

existence of $\mathrm{PbI}_{2}$ could suppress charge carrier recombination and improve the $V_{\text {oc }}$ of perovskite solar cells. ${ }^{19,20}$ Furthermore, because of the shorter $\mathrm{Pb}-\mathrm{I}$ bond length and higher bond strength, the $\mathrm{PbI}_{2}$ solubility in water is lower. As compared with perovskite, $\mathrm{PbI}_{2}$ on the surface of perovskite film is more resistant to moisture-induced degradation. ${ }^{21}$ And as a result, the water induced degradation of the perovskite solar cells will be suppressed after KI incorporation.

The crystallinity of the perovskites was analysed by XRD and the results are shown in Fig. 3. The red curve (a) and the black curve (b) represent the crystal structure of the KI incorporated and control films, respectively. It is found that both mixed perovskite films show a perovskite phase ( $\alpha$-phase), rather than a non-perovskite phase ( $\delta$-phase), regardless of the of the incorporating ions. This is consistent with the observation in other works..$^{22,23}$ The main peaks at $14.04^{\circ}, 19.94^{\circ}, 24.52^{\circ}, 28.3^{\circ}$, $31.74^{\circ}$ and $40.51^{\circ}$ are assigned to the (001), (011), (111), (002), (012) and (022) crystal planes. Peaks marked by \# and * are the diffraction peaks of the $\mathrm{PbI}_{2}$ and the substrate. After KI incorporation, the $\mathrm{PbI}_{2}$ peak is obviously suppressed, which indicates that more perovskite phase is formed. Meanwhile, the apparent enhancement of the peaks of (001) and (002) suggests good crystallinity of the tetragonal phase perovskite after incorporating. ${ }^{17}$ The better crystallinity suggests fewer defects in KI incorporated perovskite film. 


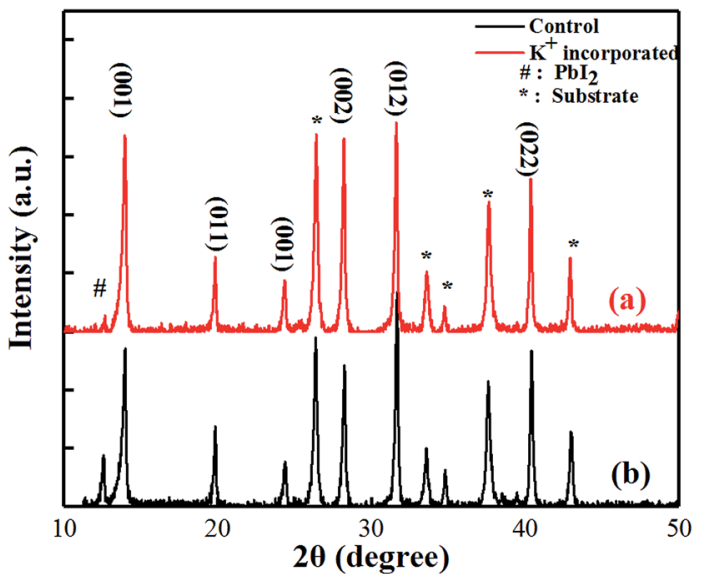

Fig. 3 XRD patterns of (a) $\mathrm{K}^{+}$incorporated and (b) control perovskite films

The KPFM was performed to measure the contact potential difference (CPD) on the surface of the film to further verify the effect of KI incorporation on GBs. The sample structure for KPFM measurement was glass $/ \mathrm{FTO} / \mathrm{SnO}_{2} / \mathrm{c}-\mathrm{TiO}_{2} / \mathrm{mp}-\mathrm{TiO}_{2} /$ perovskite. KPFM is a derivative based on atomic force microscopy with high resolution $(<10 \mathrm{~nm})$, which can get the surface topography and CPD images simultaneously, quantify local charge generation and separation within the film. The KPFM can provide a feasible approach to identify grain interiors and GBs. Fig. 4 shows the topographic images of perovskites and the corresponding KPFM results over the surface of the samples. Fig. 4a and c are the surface morphology of the perovskites and one may find that after $\mathrm{K}^{+}$incorporating, the grain size of perovskite is significantly increased, which is consistent with the SEM images. Moreover, Fig. 4a and c show that the arithmetic mean roughness $\left(R_{\mathrm{a}}\right)$ and root mean square roughness $\left(R_{\mathrm{q}}\right)$ of the film incorporated with $\mathrm{K}^{+}$are $20.4 \mathrm{~nm}$ and $26.0 \mathrm{~nm}$ respectively, which are both smaller than that of the control film (27.5 nm and $34.8 \mathrm{~nm}$, respectively). This suggests that KI incorporation effectively modifies and smoothens the surface of the perovskite. This might be contributing to the performance improvement of the corresponding devices.

Fig. $4 \mathrm{~b}$ and d show the KPFM results over the surface of the perovskites, which are obtained at the same time while measuring the surface morphology. As shown in Fig. 4a, the grain size is small, and the GBs of the control sample are not clear. And as a result, the change of CPD in Fig. $4 \mathrm{~b}$ is not obvious. On the contrary, in case of KI incorporated sample, as shown in Fig. 4c, a significant increase in the grain size could be observed, which makes the GBs clearly visible. One may notice that the CPD at the GBs in Fig. $4 d$ is smaller than that at the grain interiors. The possible reason of the decreased CPD could be the increased built-in potential that induced by the interstitial or vacancy defects at the GBs. It has been confirmed that the defects at GBs are mainly associated with iodine in perovskites. With the presence of $\mathrm{K}^{+}$, the iodine atoms are partially replaced by bromine, immobile $\mathrm{KBr}$ compounds would be generated. The charge trapping induced by the defect states at

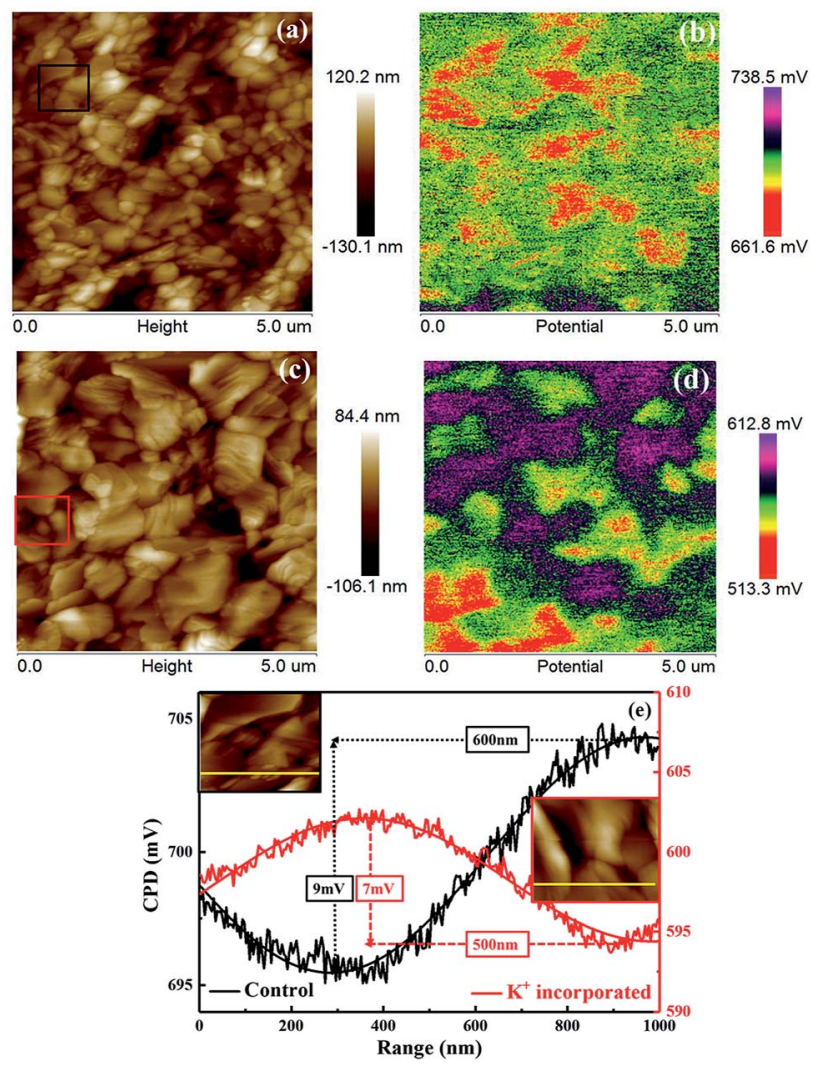

Fig. 4 Atomic force microscopy (AFM) and contact potential difference (CPD) images of ( $a$ and $b$ ) control and ( $c$ and $d$ ) incorporated perovskite films. (e) CPD distribution of control and $\mathrm{K}^{+}$incorporated samples across the GBs in black and red squares in (a) and (c).

the GBs will then be eliminated. ${ }^{24}$ In Fig. 4e, we plotted the CPD distribution of GBs corresponding to the black and red squares in Fig. 4a and c and we found that both of them consistently show the Gaussian distribution (the two yellow lines in Fig. 4e are the specific locations selected). The barrier of the carrier crossing the GB is reflected by the trough value, and the full width at half maximum (FWHM) value of the plot can be attributed to the depletion range of the GB. ${ }^{25}$ From Fig. 4e, we can see that after KI incorporation, the trough value reduced from $9 \mathrm{mV}$ to $7 \mathrm{mV}$ and FWHM reduced from $600 \mathrm{~nm}$ to $500 \mathrm{~nm}$. After KI incorporation, the depletion region of the GB is narrowed and the barrier height for the carriers to transport across the GB is reduced, which ensure efficient transportation of charge carriers.

Fig. 5 displays the steady state photoluminescence (PL) and time-resolved photoluminescence (TRPL) spectra of perovskite films. According to the PL spectra, the relative intensities of the photo-generated carrier radiation recombination and nonradiative recombination in the film can be intuitively understood. ${ }^{26}$ In order to exclude the effect of the electron transport layer on the test results, we also measured PL and TRPL of the perovskites deposited on bare glass substrates marked as control/ $\mathrm{K}^{+}$incorporated film on quartz. After KI incorporation, the band gap $\left(E_{\mathrm{g}}\right)$ of the perovskite decreases from $1.62 \mathrm{eV}$ to $1.61 \mathrm{eV}$ due to the small ionic radius of $\mathrm{K}^{+}$. As shown in Fig. 5a, 

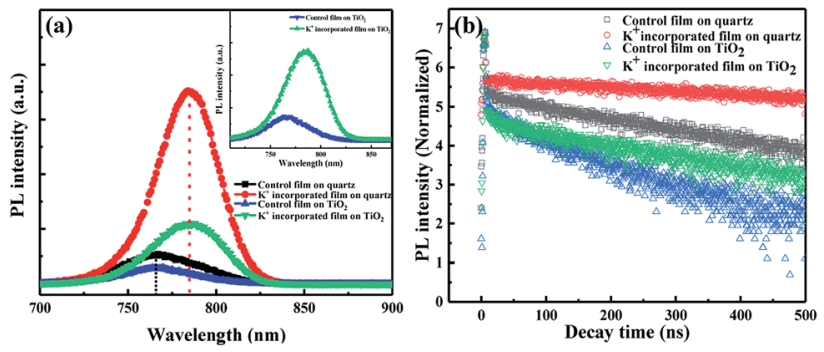

Fig. 5 (a) Steady state photoluminescence and (b) time resolved photoluminescence charts of perovskite films.

the KI-incorporated film exhibits stronger PL peak intensity whether it contains an electron transport layer (ETL) or not (the inset of Fig. $5 \mathrm{a}$ is the PL patterns of perovskites deposited on $\mathrm{TiO}_{2}$ ). For the film deposited on glass substrate, the PL intensity of KI incorporated (red) sample is ten times stronger than that of control one (black), which indicates that the incorporation of KI greatly inhibits non-radiative trap-assisted recombination due to the reduced internal defect states in perovskite thin films.

The charge carrier recombination was then further studied by using TRPL. The PL decay curves of the perovskites are shown in Fig. $5 \mathrm{~b}$ and the derived lifetimes are summarized in Table 1 . The decay curve is fitted by a double exponential function model: $I_{\mathrm{PL}(t)}=A+B_{1} \exp \left(-t / \tau_{1}\right)+B_{2} \exp \left(-t / \tau_{2}\right)$, where $t$ is time, $I_{\mathrm{PL}(t)}$ is the time-dependent PL strength, and $B_{1} / B_{2}$ is the amplitude of the attenuation component $\tau_{1} / \tau_{2}{ }^{27}$ It contains a fast decay $\left(\tau_{1}\right)$ and a slow decay process $\left(\tau_{2}\right)$. For perovskites, the $\tau_{1}$ indicates surface recombination and it is generally believed to be not obviously correlated with the device performance. Therefore, we will not discuss about it in details. ${ }^{9,28}$ On the contrary, $\tau_{2}$ is considered as the radiation recombination in bulk of perovskite before the free carriers are collected. ${ }^{29}$ In Fig. $5 \mathrm{~b}$, the red and black curves are TRPL of incorporated and control perovskite films directly deposited on quartz glass, revealing that $\tau_{2}$ increases from 281.9 to $1186 \mathrm{~ns}$ after KI incorporation. This suggests effective suppression of the recombination induced by defect states in the KI incorporated perovskite film. The elimination of the trap states in the perovskite after KI incorporation is ascribed to the occupation of the halide vacancy defects by the excess $\mathrm{I}^{-} .^{30}$ At the same time, $\tau_{1}$ and $\tau_{2}$ in both perovskites deposited on ETL coated substrates before and after KI incorporation are decreased in comparison with that deposited on bare glass. This reflects efficient transport of electrons from the perovskite layer to the

Table $1 \mathrm{PL}$ and TRPL tested parameters

\begin{tabular}{lllc}
\hline & $E_{\mathrm{g}}(\mathrm{eV})$ & $\tau_{1}(\mathrm{~ns})$ & $\tau_{2}(\mathrm{~ns})$ \\
\hline Control film on quartz & 1.62 & 0.875 & 281.9 \\
$\mathrm{~K}^{+}$incorporated film on quartz & 1.58 & 0.957 & 1186 \\
Control film on $\mathrm{TiO}_{2}$ & 1.62 & 0.509 & 123.8 \\
$\mathrm{~K}^{+}$incorporated film on $\mathrm{TiO}_{2}$ & 1.58 & 0.752 & 239.6
\end{tabular}

ETL. It is worth noting that though the lifetime of incorporated one (239.6 ns) is still higher than the control (123.8 ns), it reduced a lot compared to the original lifetime without ETL. Which not only indicates the lower defect concentration of the film itself, but also suggests an improved charge transport capability at the interface of absorber/ETL after incorporating.

The recombination mechanism of perovskite solar cells was then investigated by using temperature-dependent currentvoltage (JVT) measurement. Fig. 6a and b show the fitted line of $V_{\text {oc }}$ as a function of $T$ during heating and cooling processes, respectively. By fitting the $J-V$ curves with the ideal diode eqn (1) and $J_{0}$ can be expressed as eqn (2). According to the activation energy determined from the $V_{\mathrm{oc}}$-temperature $\left(V_{\mathrm{oc}}-T\right)$ curve by the eqn (3):

$$
\begin{gathered}
V_{\mathrm{oc}}=\frac{A k T}{q} \ln \left(\frac{J_{\mathrm{sc}}}{J_{0}}\right) \\
J_{0}=J_{00}\left[\exp \left(\frac{-E_{\mathrm{a}}}{A k T}\right)\right] \\
V_{\mathrm{oc}}=\frac{E_{\mathrm{a}}}{q}-\frac{A k T}{q} \ln \frac{J_{00}}{J_{\mathrm{sc}}}
\end{gathered}
$$

where $E_{\mathrm{a}}, A, k, T, J_{0}, J_{00}$ and $J_{\mathrm{sc}}$ are respectively represent activation energy associated with recombination in solar cells, diode ideal factor, Boltzmann constant, temperature, the diode current, reverse saturation current and short circuit density. ${ }^{31,32}$ The activation energy could distinguish the dominant recombination path between the interface and the bulk, and an activation energy smaller than the bandgap often implies the significance of interfacial recombination. The value of the activation energy was estimated by extrapolating the line from the $V_{\mathrm{oc}}-T$ curve to $T=0 \mathrm{~K}$, thus avoiding fitting errors. In case of a traditional semiconductor, due to the electron-photon interaction and lattice thermal expansion induced changes in the energy band, the bandgap shows a negative temperature dependence. ${ }^{33}$ However, for organic-inorganic hybrid perovskites, opposite to the traditional semiconductors, with the increased temperature, their bandgaps keep increasing within a certain range (270-420 K) and the temperature coefficient of perovskite material is $\mathrm{d} E_{\mathrm{g}} / \mathrm{d} T=0.3 \mathrm{meV} \mathrm{K}{ }^{-1} \cdot{ }^{34}$

We measured the temperature dependent light $J-V$ of the devices in a temperature cycle of 300-360-300 K. The results of
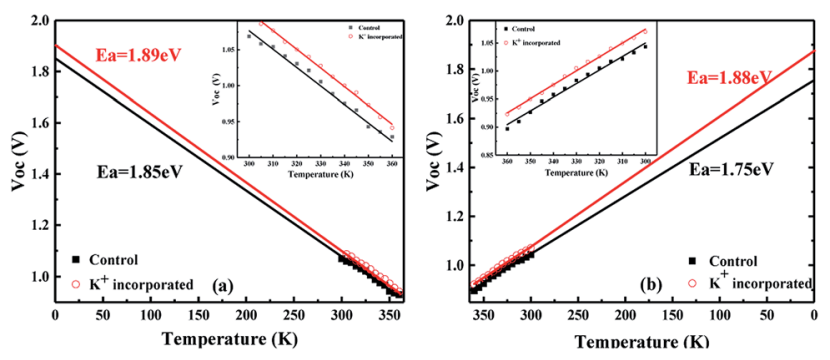

Fig. 6 Temperature-dependent current-voltage image $\left(V_{o c}-T\right)$ of devices at AM1.5, the solid lines are the linear fitting of the measured points. (a) and (b) are the heating and cooling processes, respectively. 
the heating and cooling processes for both devices are shown in Fig. 6. In heating process, the $V_{\text {oc }}$ are linearly correlated with temperature. In both devices, $V_{\text {oc }}$ follows a straight line for temperature and the extrapolation of the activation energy is higher than the $E_{\mathrm{g}}$ of the perovskite calculated according to the temperature coefficient. Furthermore, the activation energy of the device after incorporating $\mathrm{KI}(1.89 \mathrm{eV})$ is greater than that of control one $(1.85 \mathrm{eV})$. These indicate that there are fewer defects inside the incorporated perovskite film, better contact with the functional layers and the internal recombination of carriers is much larger than the interfacial recombination. ${ }^{31}$ All of the aforementioned factors contribute to the improved device performance. Fig. $6 \mathrm{~b}$ shows the temperature dependent $V_{\mathrm{oc}}$ when the sample is cooled down from $360 \mathrm{~K}$ to $300 \mathrm{~K}$. It can be found that the $V_{\mathrm{oc}}$ of the cell incorporated with KI can be normally restored to the original state, and thus the derived activation energy is hardly changed. However, on the contrary, the $V_{\text {oc }}$ of the control device cannot be restored to the initial state with a reduced activation energy from $1.85 \mathrm{eV}$ to $1.75 \mathrm{eV}$. This is attributed to the fact that incorporating KI not only reduces the interfacial recombination, but also improves the thermal stability of the cell. As can be seen in Fig. S3, $\uparrow$ we have performed the temperature-dependent IV measurements on over 5 sets of control/incorporated perovskite solar cells and found that results were well reproducible. The activation energies of the incorporated samples were between 1.89-1.96 eV, and those of the control ones were between 1.82-1.87 eV.

Fig. 7 is the forward and reverse scan of the $J-V$ curves for specific devices which possess the photovoltaic parameters (summarized in Table 2) close to the average values of each batch. From Fig. 7 and Table 2, we can find that the $V_{\text {oc }}$ and FF are significantly improved after KI incorporation, which indicates that the non-radiative trap-assisted recombination inside the cell is greatly suppressed and as a result, a significant optimization of the PCE from $17.3 \%$ to $19.5 \%$ was witnessed. Apart from PCE, hysteresis factor is another important parameter for perovskite solar cells. The hysteresis factor is defined as $\left(\mathrm{PCE}_{\text {Reverse }}-\mathrm{PCE}_{\text {Forward }}\right) / \mathrm{PCE}_{\text {Reverse }}$, and it is a parameter that describes the magnitude of hysteresis effect. ${ }^{35}$ From the $J-V$ curves of the both devices shown in Fig. 7, one may notice that not only the efficiency of the cell is increased, but also the

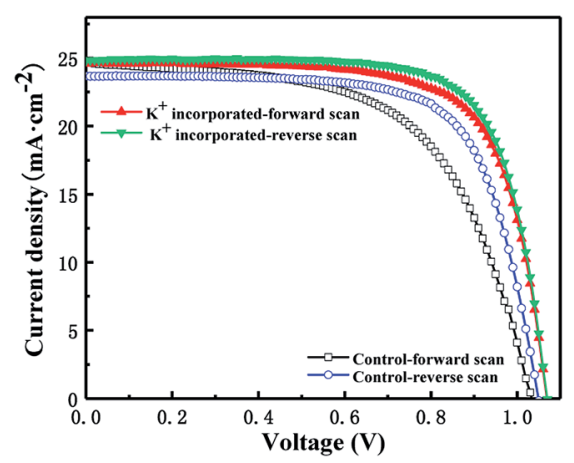

Fig. 7 Current density-voltage $(J-V)$ curves for representative devices of incorporated and control perovskite solar cells.
Table 2 The specific parameters of the test cells

\begin{tabular}{lccccc}
\hline & & & & & $\begin{array}{l}\text { Hysteresis } \\
\text { factor }\end{array}$ \\
\hline $\mathrm{K}^{+}$incorporated-FS & 24.6 & 1.07 & 72.1 & 19.0 & $\mathbf{0 . 0 3}$ \\
$\mathrm{K}^{+}$incorporated-RS & 24.8 & 1.07 & 73.6 & 19.5 & \\
Control-FS & 24.7 & 1.03 & 58.9 & 15.1 & $\mathbf{0 . 1 3}$ \\
Control-RS & 23.7 & 1.05 & 69.8 & 17.3 &
\end{tabular}

difference between the forward scan and the reverse scan is reduced after KI incorporation and the hysteresis factor decreased from 0.13 to 0.03 (see Table 2). It has been reported that grain size and GBs, ${ }^{36}$ ion migration, ${ }^{37}$ band gap misalignment ${ }^{38,39}$ and defects state $\mathbf{4 0 , 4 1}^{\mathbf{3}}$ are the possible causes of the hysteresis effect. Therefore, in our case, the increased grain size, reduced defects at the GBs and better contact at the interface for KI incorporated device are contributing to a suppressed hysteresis.

Currently, the stability is the most critical factor that limits the commercial application of perovskite solar cells. In order to investigate the stability of the perovskite solar cells, the unencapsulated devices were exposed to ambient air with a temperature of $25{ }^{\circ} \mathrm{C}$ and humidity of $40 \%$. Except for carrying out illuminated $J-V$ characteristics, the devices were kept in the dark.

Therefore, we can intuitively detect the dark state stability of perovskite solar cells and eliminate the effects of light and temperature. In Fig. S1 of ESI, $\uparrow$ there are five $J-V$ curves of control and KI incorporated devices, and we choose one of them to monitor its degradation process. From the variation of normalized PCE during the degradation process, as shown in Fig. 8, one may find that for control device, the efficiency drops rapidly to $80 \%$ within $200 \mathrm{~h}$. In contrast, it takes over 1000 hours for the KI incorporated device to decay to $80 \%$ of its initial value. The absolute values of $J_{\mathrm{sc}}, V_{\mathrm{oc}}, \mathrm{FF}$ and PCE for the lifetime measurements can be seen in ESI Fig. S2. $\uparrow$ This suggests that KI incorporation is also an effective strategy to improve the longterm stability of perovskite solar cells and has an important indication in pushing forward the commercialization of organic-inorganic halide hybrid PV cells as well as modules.

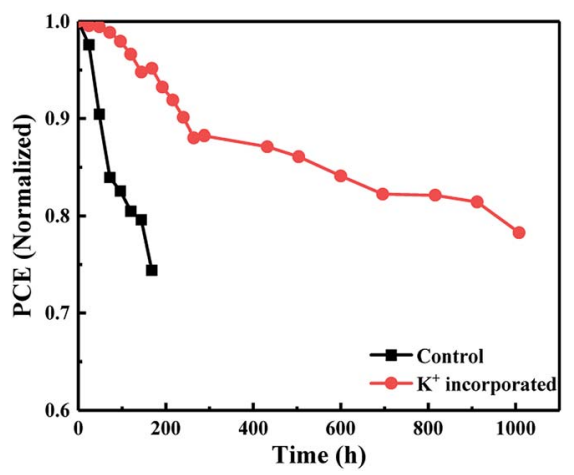

Fig. 8 The shelf lifetime trace of the control and incorporated devices (the temperature is $25^{\circ} \mathrm{C}$ and the relative humidity is $40 \%$ ). 


\section{Conclusions}

By thoroughly investigating the properties of pristine and KI incorporated perovskite thin films/devices, the passivation mechanism of KI was illustrated. The enhanced PL peak and improved carrier lifetime of KI incorporated films suggests that the defects within the film were significantly passivated to inhibit non-radiative recombination. The KPFM results suggest that the narrowed depletion region of the grain boundary and the decreased barrier height are beneficial for the charge transportation within the films. JVT characterization proved that the internal recombination is predominant in both devices and the interface between the perovskite absorption layer and the interface of perovskite/electron transport was also significantly passivated after $\mathrm{KI}$ incorporation. The $\mathrm{KI}$ incorporation is therefore verified to be an effective solution to passivate not only the defects within the perovskite films, but also the interface of the devices. The KI passivation significantly improves the device performance (average PCE improved from $17.9 \%$ to $19.3 \%$ and hysteresis factor decreased from 0.13 to 0.03 ) and long-term stability ( $\tau_{80}$ increased from less than 200 hours to over 1000 hours) of the perovskite solar cells.

\section{Conflicts of interest}

There are no conflicts to declare.

\section{Acknowledgements}

This work was financially supported by the Science and Technology Program of Sichuan Province (No. 2017GZ0052) and the Fundamental Research Funds for the Central Universities (No. YJ201722).

\section{References}

1 National Renewable Energy Laboratory, Best research-cell efficiencies, https://www.nrel.gov/pv/assets/pdfs/bestresearch-cell-efficiencies-190416.pdf, visited in April 2019.

2 A. Zhumekenov, M. Saidaminov, M. Haque, E. Alarousu, S. Sarmah, B. Murali, I. Dursun, X.-H. Miao, A. Abdelhady, T. Wu, O. Mohammed and O. Bakr, ACS Energy Lett., 2016, 1, 32-37.

3 Q. Dong, Y. Fang, Y. Shao, P. Mulligan, J. Qiu, L. Cao and J. Huang, Science, 2015, 347, 967-970.

4 S.-H. Turren-Cruz, M. Saliba, M. Mayer, H. JuárezSantiesteban, X. Mathew, L. Nienhaus, W. Tress, M. Erodici, M.-J. Sher, M. Bawendi, M. Grätzel, A. Abate, A. Hagfeldt and J.-P. Correa-Baena, Energy Environ. Sci., 2018, 11, 78-86.

5 A. Bowring, W. Tan, A. Meng, M. McGehee and P. McIntyre, ACS Appl. Mater. Interfaces, 2018, 10, 5485-5491.

6 M. Yang, Y. Zeng, Z. Li, D. Kim, C.-S. Jiang, J. Lagemaat and K. Zhu, Phys. Chem. Chem. Phys., 2017, 19, 5043-5050.

7 S. Yang, J. Dai, Z. Yu, Y. Shao, Y. Zhou, X. Xiao, X. Zeng and J. Huang, J. Am. Chem. Soc., 2019, 141, 5781-5787.
8 T. Niu, J. Lu, R. Munir, J. Li, D. Barrit, X. Zhang, H. Hu, Z. Yang, A. Amassian, K. Zhao and S. Liu, Adv. Mater., 2018, 30, e1706576.

9 D. Lee, J. Yun, J. Kim, A. Soufiani, S. Chen, Y. Cho, X. Deng, J. Seidel, S. Lim, S. Huang and A. Ho-Baillie, ACS Energy Lett., 2018, 3, 647-654.

10 D.-Y. Son, S.-G. Kim, J.-Y. Seo, S.-H. Lee, H. Shin, D. Lee and N.-G. Park, J. Am. Chem. Soc., 2018, 140, 1358-1364.

11 Z. Tang, T. Bessho, F. Awai, T. Kinoshita, M. Maitani, R. Jono, T. Murakami, H. Wang, T. Kubo, S. Uchida and H. Segawa, Sci. Rep., 2017, 7, 12183.

12 M. Saliba, T. Matsui, K. Domanski, J.-Y. Seo, A. Ummadisingu, S. Zakeeruddin, J.-P. Correa-Baena, W. Tress, A. Abate, A. Hagfeldt and M. Grätzel, Science, 2016, 354, 206-209.

13 T. Duong, H. Mulmudi, H. Shen, Y. Wu, C. Barugkin, Y. Mayon, H. Nguyen, D. Macdonald, J. Peng, M. Lockrey, W. Li, Y.-B. Cheng, T. White, K. Weber and K. Catchpol, Nano Energy, 2016, 30, 330-340.

14 M. Saliba, T. Matsui, J.-Y. Seo, K. Domanski, J.-P. CorreaBaena, M. Nazeeruddin, S. Zakeeruddin, W. Tress, A. Abate, A. Hagfeldt and M. Grätzel, Energy Environ. Sci., 2016, 9, 1989-1997.

15 M. Deepa, M. Salado, L. Calio, S. Kazim, S. Shivaprasadc and S. Ahmad, Phys. Chem. Chem. Phys., 2017, 19, 4069-4077.

16 T. Bu, X. Liu, Y. Zhou, J. Yi, X. Huang, L. Luo, J. Xiao, Z. Ku, Y. Peng, F. Huang, Y.-B. Cheng and J. Zhong, Energy Environ. Sci., 2017, 10, 2509-2515.

17 G. Zheng, C. Zhu, J. Ma, X. Zhang, G. Tang, R. Li, Y. Chen, L. Li, J. Hu, J. Hong, Q. Chen, X. Gao and H. Zhou, Nat. Commun., 2018, 9, 2793.

18 J.-J. Li, J.-Y. Ma, Q.-Q. Ge, J.-S. Hu, D. Wang and L.-J. Wan, ACS Appl. Mater. Interfaces, 2015, 7, 28518-28523.

19 T. Zhang, N. Guo, G. Li, X. Qian and Y. Zhao, Nano Energy, 2016, 26, 50-56.

20 Y. Kim, N. Jeon, J. Noh, W. Yang, J. Seo, J. Yun, A. Ho-Baillie, S. Huang, M. Green, J. Seidel, T. Ahn and S. Seok, Adv. Energy Mater., 2016, 6, 1502104.

21 Q. Wang, B. Chen, Y. Liu, Y. Deng, Y. Bai, Q. Dong and J. Huang, Energy Environ. Sci., 2017, 10, 516-522.

22 L. Zuo, H. Guo, D. deQuilettes, S. Jariwala, N. Marco, S. Dong, R. DeBlock, D. Ginger, B. Dunn, M. Wang and Y. Yang, Sci. Adv., 2017, 3, e1700106.

23 L. Zuo, S. Dong, N. Marco, Y.-T. Hsieh, S.-H. Bae, P. Sun and Y. Yang, J. Am. Chem. Soc., 2016, 138, 15710-15716.

24 F. Zheng, W. Chen, T. Bu, K. Ghiggino, F. Huang, Y. Cheng, P. Tapping, T. Kee, B. Jia and X. Wen, Adv. Energy Mater., 2019, 1901016.

25 C. Li, G. Chen, W. Wang, J. Zhang, L. Wu, X. Hao and L. Feng, J. Mater. Sci.: Mater. Electron., 2018, 29, 2071820725.

26 T. Du, C. Burgess, C.-T. Lin, F. Eisner, J. Kim, S. Xu, H. Kang, J. Durrant and M. McLachlan, Adv. Funct. Mater., 2018, 28, 1803943.

27 G. Niu, H. Yu, J. Li, D. Wang and L. Wang, Nano Energy, 2016, 27, 87-94. 
28 Q. Dong, Z. Wang, K. Zhang, H. Yu, P. Huang, X. Liu, Y. Zhou, N. Chen and B. Song, Nanoscale, 2016, 8, 55525558.

29 Q. Dong, K. Zhang, H. Yu, P. Huang, X. Liu, Y. Zhou, N. Chen and B. Song, Nanoscale, 2016, 8, 5552-5558.

30 M. Abdi-Jalebi, Z. Andaji-Garmaroudi, S. Cacovich, C. Stavrakas, B. Philippe, J. Richter, M. Alsari, E. Booker, E. Hutter, A. Pearson, S. Lilliu, T. Savenije, H. Rensmo, G. Divitini, C. Ducati, R. Friend and S. Stranks, Nature, 2018, 555, 497-501.

31 D. Abou-Ras, T. Kirchartz and U. Rau, Advanced Characterization Techniques for Thin Film Solar Cells, WileyVCH, 2011.

32 C. Ding, Y. Zhang, F. Liu, Y. Kitabatake, S. Hayase, T. Toyoda, K. Yoshino, T. Minemoto, K. Katayam and Q. Shen, Nano Energy, 2018, 53, 17-26.

33 P. Dey, J. Paul, J. Bylsma, D. Karaiskaj, J. M. Luther, M. C. Beard and A. H. Romero, Solid State Commun., 2013, 165, 49-54.
34 O. Dupré, R. Vaillon and M. A. Green, Sol. Energy Mater. Sol. Cells, 2015, 140, 92-100.

35 Z. Li, J. Tinkham, P. Schulz, M. Yang, D. Kim, J. Berry, A. Sellinger and K. Zhu, Adv. Energy Mater., 2017, 7, 1601451. 36 Y. Shao, Z. Xiao, C. Bi, Y. Yuan and J. Huang, Nat. Commun., 2014, 5, 5784 .

37 C. Li, A. Guerrero, Y. Zhong and S. Huettner, J. Phys.: Condens. Matter, 2017, 29, 193001.

38 Y. Ju, S. Park, H. Han and H. Jung, RSC Adv., 2019, 9, 73347337.

39 H. Li, Y. Xue, B. Zheng, J. Tian, H. Wang, C. Gao and X. Liu, RSC Adv., 2017, 7, 30422-30427.

40 J.-W. Lee, S.-G. Kim, S.-H. Bae, D.-K. Lee, O. Lin, Y. Yang and N.-G. Park, Nano Lett., 2017, 17, 4270-4276.

41 J. Azpiroz, E. Mosconi, J. Bisquert and F. Angelis, Energy Environ. Sci., 2015, 8, 2118-2127. 